\title{
Atmospheric sodium monitor for Laser Guide Star Adaptive Optics
}

\author{
N. Ageorges ${ }^{1}$ and N. Hubin ${ }^{2}$ \\ 1 National University of Ireland, Galway, Physics Department, Galway, Ireland \\ e-mail: nancy@physics.ucg.ie \\ 2 European Southern Observatory, Karl Schwarzschildstraße 2, D-85748 Garching, Germany \\ e-mail: nhubin@eso.org
}

Received May 17, 1999; accepted April 3, 2000

\begin{abstract}
Laser Guide Star Adaptive Optics (AO) makes use of a laser to excite the mesospheric sodium ( $\mathrm{Na}$ ) layer and thereby creates an artificial star that can be used as reference for the AO wavefront sensor. The intensity of the created star depends strongly on the amount of $\mathrm{Na}$ present in the atmosphere. Experiments to measure the column density and details of the excitation and scattering properties of sodium atoms in the corresponding atmospheric layer are thus very important to refine the design parameters of laser and assess the power requirement.

This article reviews the current knowledge on the mesospheric sodium derived from both astronomical and atmospherical studies. It then presents possible methods to study the sodium layer characteristics relevant for adaptive optics laser guide star systems.
\end{abstract}

Key words: instrumentation: adaptive optics instrumentations: miscellaneous - methods: observational

\section{Introduction}

Most recent Laser Guide Star (LGS) systems for astronomical Adaptive Optics (AO) makes use of mesospheric scattering of a sodium laser (at the Multi-Mirror Telescope (MMT), e.g. Lloyd-Hart et al. 1998, at Lick Observatory, e.g. Olivier et al. 1996, or Calar Alto, e.g. Davies et al. 1998), whereby an artificial reference star, which can be used as reference for the AO wavefront sensor, is created at the altitude of the mesospheric sodium layer (around $95 \mathrm{~km}$ in average).

The optimisation of adaptive optics systems using $\mathrm{Na}$ guide stars requires a detailed knowledge of the temporal and spatial characteristics of the atmospheric Na layer on

Send offprint requests to: N. Ageorges,

e-mail: nageorge@eso.org both long and short-term scale. Experiments to measure the column density and details of the excitation and scattering properties of sodium atoms in the mesospheric layer are thus very important to refine the design parameters of lasers for laser guide star systems. Indeed the intensity of the artificial star depends strongly on the amount of sodium present in the atmosphere.

Seasonal variations of both the column density and the altitude of the mesospheric $\mathrm{Na}$, which impact respectively on the necessary LGS power and its focus altitude, can be studied in a statistical way. The daily and hourly variations also bring information on the variation of the averaged column density and height, but on short timescale and therefore become important for queue scheduling of LGS-AO observations, as planed e.g. at the European Southern Observatory (ESO) Very Large Telescope (VLT).

Study of the atmospheric sodium is considered here under two different aspects:

- the statistical study of the general properties of current and prospective observing sites;

- the direct monitoring of short term variations in the mesospheric sodium, on sites housing large telescope projects.

This paper aims at reviewing the current knowledge on atmospheric sodium and its impact on laser guide star adaptive optics system operations. The sodium characteristics that have a direct impact on LGS AO observations are presented in Sect. 2. Section 3 will illustrate our on-going work among statistical studies. Section 4 concentrates on the monitoring problem and states some possibilities for "on-line" study of mesospheric sodium.

\section{Atmospheric sodium properties relevant to LGS-AO}

\subsection{Introduction}

Since the discovery of the presence of alkaline metals in atomic form at the altitude of the mesopause 
(Slipher 1929; Bricard et al. 1949), the origin and behaviour of this layer of atoms has been the subject of numerous experimental and theoretical researches; studies of the alkali metals, atmospheric sounding, studies of a meteoritic metallic layer at $100 \mathrm{~km}$, radar and lidar studies of gravity waves, and, since the late 70's, studies of the sporadic sodium layers and measurements to explain the seasonal and meridional variations in the sodium layer height, thickness and density. Because of its relatively high concentration in meteors and its large resonant backscatter cross section, $\mathrm{Na}$ is the easiest metallic species and the easiest of the meteoric layer atoms to observe by resonance fluorescence lidar techniques (Bowman et al. 1969).

\subsection{Long-term variations}

All previous atmospheric studies have found that the mesospheric sodium column density has diurnal (e.g. McNutt \& Mack 1963), nocturnal and seasonal variations (see e.g. Fig. 2 of Papen et al. 1996). Moreover, lidar measurements of the sodium column densities, performed since 1969 (see e.g. Gibson \& Sandford 1971; Simonich et al. 1979 for some of the first ones at different latitude) have confirmed the previous photometric measurements (e.g. Donahue \& Blamont 1961; Hunten et al. 1964; Rees et al. 1975), which have shown that the sodium total abundance also presents seasonal variations dependent on the latitude (e.g. Jegou 1985). Once the necessary laser power has been determined from the $\mathrm{Na}$ column density seasonal minimum, these variations have little impact on LGS AO systems since in average over a year the sodium column density is, within a factor $\leq 3$ the same at all latitudes $\left(\approx 310^{9} \mathrm{~cm}^{-2}\right)$. The minimum in $\mathrm{Na}$ abundance around July in the northern hemisphere corresponds to a maximum in the southern hemisphere and inversely around January. This means that each observatory will have few months where LGS AO observations will be more performant than other months. Indeed the strongest the sodium abundance, the brightest the artificial star for a given laser power (Milonni et al. 1998), assuming there is no saturation at the sodium layer.

\subsection{Short-term variations}

\subsubsection{Introduction}

It has also been found, from the observations mentioned in 2.2, that both the averaged altitude of the mesospheric sodium varies as well as the column density (Papen et al. 1996). Some of these variations can be extremely quick due to the presence of sporadic layers, $\mathrm{Na}_{\mathrm{s}}$ (see e.g O'Sullivan et al. 2000), which are very thin $\mathrm{Na}$ layers superposed on the mean mesospheric Na layers.
They have been observed at several lidar sites, but there appears to be no significant seasonal difference in occurrence frequency or maximum sodium concentration. The formation mechanisms of these layers are not yet well-understood, but $\mathrm{Na}_{\mathrm{s}}$ layers have always appeared to be strongly latitude-dependent phenomena, having been regularly observed at both low and high latitudes but not, until recently (Nagasawa \& Abo, 1995), at mid-latitude sites (cf. Clemesha et al. 1980; von Zahn et al. 1987; von Zahn \& Hansen 1988; Gardner et al. 1988; Kwon et al. 1988). Sporadics are characterised by a full-widths at half-maximum of typically 1 to $2 \mathrm{~km}$. The ratio of maximum peak density of the sporadic layer to the density of the normal layer at the altitude of the peak sporadic layer density is typically from 3 to 5 and their values can be rarely as much as the order of 10 . The events can last from a few tens of minutes to several hours. Figure 4 of Gu et al. (1995) is a very good illustration of observation of a sporadic event. In the late 70's, Clemesha et al. (1978) and Clemesha et al. (1980) were the first to report such observations at Sao Paulo, Brazil $\left(23^{\circ} \mathrm{S}, 46^{\circ} \mathrm{W}\right)$. Later Kwon et al. (1988) found that most of the sporadics appearing above Mauna Kea (Hawaii) are located in the same two hours slot of Local Sideral Time (LST). The occurrence of sporadic layers of neutral sodium and other metals at heights around $100 \mathrm{~km}$, with thickness much less than the atmospheric scale height, is an interesting and puzzling phenomenon, which has been nicely reviewed by Clemesha (1995).

\subsubsection{Influence of sporadic layers on LGS operation}

The recognition of $\mathrm{Na}_{\mathrm{S}}$ layers is crucial for LGS-AO operation, because they cause rapid local increase in the column density and an associated displacement of the altitude's centroid of the whole layer (see e.g. Fig. 4 of Beatty et al. 1989). The effective Na layer altitude (the centroid height) moves first upwards as the sporadic layer appears and then downwards (see e.g. Fig. 2 of Senft et al. 1989). This produces a rapid focus shift for the LGS on a time scale of a few seconds to several minutes. The largest centroid variation observed in Fig. 4 of Beatty et al. (1989) produces a change of the order of $250 \mathrm{~m}$ in $30 \mathrm{~s}$. For the VLT this would mean a wavefront error of $\sigma \sim 39 \mathrm{~nm}$ rms, exceeding the NAOS (Natural guide star Adaptive Optics System - the AO system for the VLT telescopes) mean error budget, of $15 \mathrm{~nm} \mathrm{rms.}$ This also corresponds to an equivalent phase variance of $0.06 \mathrm{rad}^{2}$ at $1 \mu \mathrm{m}$ due to defocus, which is e.g. perfectly acceptable for the VLT AO system, i.e. largely within the error bars. Although not critical to most AO LGS operation, this effect has to be taken into account in the overall error budget.

A change in the Na-layer centroid height during the astronomical exposure is seen by the AO wavefront sensor, 
observing the LGS, as a defocus component in the atmospheric wavefront analysis and is automatically corrected by the AO deformable mirror, partially defocussing the object at infinity. A proposed way to solve this problem is to remember that atmospheric perturbations measured in Adaptive Optics average over a time period of about 1 min to zero (Hubin \& Noethe 1991). Using this characteristic, we propose to store the overall defocus (atmospheric + sodium layer centroid height) obtained on the LGS wavefront sensor for the deformable mirror control and to average it out over a period of $1 \mathrm{~min}$. After averaging, the sole defocus produced by the sodium profile variation remains and can be corrected by defocusing the LGS wavefront sensor. This method works only if variations of the sodium profile can be neglected under $1 \mathrm{~min}$, which has not been proven yet.

\section{Statistical study}

The statistical study aims at quantifying the average and variability of the column density of neutral sodium present in the upper atmosphere, at different observation sites, in order to establish the minimum laser power necessary for LGS-AO.

\subsection{Indirect knowledge about the atmospheric Na: Astronomical observations}

High resolution spectroscopic studies of the $\mathrm{Na} \mathrm{D}$ lines in the interstellar medium (see e.g. Welsh et al. 1990; Welsh et al. 1994) suggest a methodology whereby the mesospheric neutral sodium column density can be determined from normal astronomical observations, although there has been no systematic attempt to do so until now.

There have been, over the last decade, an increasing number of studies of the local interstellar medium (LISM), defined here as $d<200$ pc, using both optical and UV absorption lines (see reviews by Paresce 1984 and by Cox \& Reynolds 1987). Thanks to high resolution ground based observations, a large database of information exists concerning the absorption characteristics and galactic distribution of neutral interstellar sodium (Na I) gas. Specifically, this data has been gained over the past 25 years through observations of the $\mathrm{Na} I \mathrm{D}$ line doublet at $5890 \AA$ seen in the absorption spectra of galactic early-type stars (e.g. Hobbs 1978) which have no intrinsic sodium features, either in emission or absorption.

The interesting result for the problem considered here, is the fact that the dense neutral interstellar $\mathrm{NaI}$ gas is generally absent from the very local interstellar medium. Indeed no sodium absorption was detected towards any of the stars, observed by Welsh et al. (1990), with distances less than $42 \mathrm{pc}$. This means that it is possible, in principle, to measure the mesospheric sodium column density from observations of sodium absorption in early-type stars closer than $\sim 50$ pc for which there is little or no interstellar contribution (interstellar equivalent width are typically less than $10 \mathrm{~m} \AA$ ).

\subsection{Archive work}

One possibility for a statistical study of column density alone is to use data from astronomical archives. To reduce the work, sites having and/or planning to have LGS-AO have been identified from the list established in Rigaut (1997). In the second part of the work we set out to identify existing archive data for those particular sites that could be used for the statistical study.

It results from this research that there is little high resolution spectroscopic data at the interesting wavelengths in the available data archives. Moreover, the existing data are sparse and spread over time and geographical location. Hence there is no reasonable possibility to make a meaningful statistical study of the atmospheric sodium density above any particular observatory. In the long term a dedicated archive will have to be created.

\subsection{What is currently being done}

This section will concentrate only on the collaborative efforts of ESO and the National University of Ireland, Galway (NUIG) concerning the problem of measuring the column density of atmospheric sodium with existing intruments, as preparatory work for the laser guide star adaptive optics system planned for the VLT at Paranal. In this framework ESO and NUIG are collaborating within the Training and Mobility of Reasearcher (TMR) program on "Laser guide star for $8 \mathrm{~m}$ class telescopes", funded by the European Commission.

Since it became apparent, from studies of the data archives, that no data exist concerning the abundance of sodium above the Chilean observatories, we decided to make, specifically for this purpose, high resolution spectroscopic measurements of selected stars at ESO La Silla. The only other measurements existing in South America have been done in Brazil by Clemesha et al. (1992). No LGS-AO is planned for La Silla, but for Cerro Paranal, which is $\approx 700 \mathrm{~km}$ northward $\left(\Delta \ell=4^{\circ} 35^{\prime}\right)$. The $\mathrm{Na}$ abundance may, of course, differ somewhat between La Silla and Paranal, however, in the absence of specific measurements at Paranal itself, these observations will give a general idea of the amount of sodium present. They are also interesting for comparison with the results from the Brazilian observations.

The observations aimed at determining the absolute averaged sodium density in the atmosphere above La Silla and the night and day-to-day variations. The data were obtained during one and a half night granted Director 
Discretionary Time in July 1998, on the ESO Multi Mode Instrument (EMMI, Dekker et al. 1986) at the New Technology Telescope (NTT).

The sources, selected from Welsh et al. (1994), are unreddened early-type stars closer than 50 pc. This allows to observe pure atmospheric absorption spectra in the vicinity of the $\mathrm{NaD}$ doublet free from interstellar absorption and stellar features in absorption or emission. The stars are of type A \& B, i.e. with flat spectrum, and have been selected to have $m_{V}<10$, for high incoming flux. Ideally one would like to have short integration times to detect the sporadics and quick variation of the atmospheric sodium. However this is currently impossible for high resolution spectroscopic measurements.

\subsubsection{Observations}

The minimum resolution necessary to resolve the atmospheric sodium D lines from nearby contaminating water line is of 50000. This thus implied the choice of the EMMI echelle grating \#14.

To measure the $\mathrm{D}_{1}$ equivalent width above a $3 \sigma$ level, ideally one would like a signal to noise ratio (SNR) of 1000 , with:

$$
S N R=\frac{D Q E \times \delta \lambda \times N_{\mathrm{obj}} \times t}{\sqrt{\left(D Q E \times \delta \lambda \times N_{\mathrm{obj}} \times t\right)+\left(D Q E \times \delta \lambda \times N_{\mathrm{sky}} \times t\right)+D N^{2}}}
$$

where $D Q E$ is the dectector quantum efficiency ( 0.65 for EMMI), $\delta t$ the width of a spectral resolution element (in $\AA$ ) , $N_{\text {obj }}$ the number of photons per second per $\AA$ per spatial resolution element incident on the detector from the object, $N_{\text {sky }}$ same as $N_{\text {obj }}$ but for photons from the sky, $t$ the integration time (in sec) and $D N$ the detector noise per resolution element. Since we are considering only bright stars $\left(m_{V}<6\right)$, the sky contribution is negligible and will thus not be considered in this calculation.

The detector of interest is the CCD\#36, which has a read_out_noise of 5 electrons/pixel and a dark of 1.7 electrons/pixel/hour; so that $D N=N \times 5+(1.7 / 3600) \times t$, with $t$ the integration time and $N$ the total number of exposures. This term is however negligible since the observations are dominated by the photon noise. It is thus reasonable to approximate the signal to noise ratio by:

$S N R \approx \sqrt{\left(D Q E \times \delta \lambda \times N_{\mathrm{obj}} \times t\right)}$.

The slit has been selected to have $1^{\prime \prime}$ width and $6.5^{\prime \prime}$ length on the sky, corresponding to an expected resolution of 60000.

For EMMI at the NTT and the echelle grating used, Dekker et al. (1994) quote the arrival of 1 photon $/ \AA / s$ at $5500 \AA$ for a $m_{V}=16.6$ star, at an airmass of 1 . For a $m_{V}=4.7$ star, a pixel scale of $0.27^{\prime \prime}$, and taking the quantum efficiency of the detector into account, one finds 403.3 electrons $/ \AA / \mathrm{s}$ arriving on the detector. Since the linearity of the detector is 50000 ADUs, and the gain 2.2 electrons/ADU, in this case, one can integrate for $\approx 273 \mathrm{~s}$,

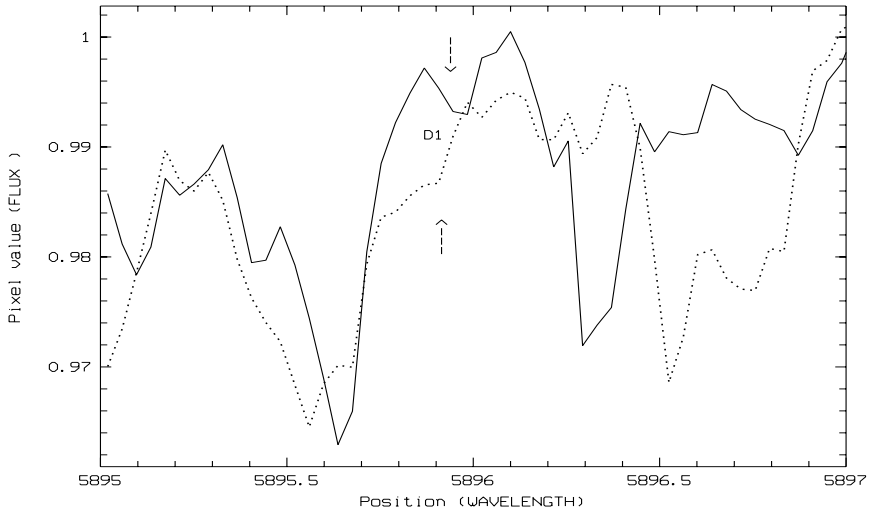

Fig. 1. Spectrum of $\theta$ Cap (full line). It is the sum of 28 spectra corresponding to $5870 \mathrm{~s}$. of integration on source. The $\mathrm{D}_{1}$ line is clearly resolved at $5895.96 \AA$. Overplotted (dotted line) is the spectrum of $\beta \mathrm{Lib}$, corresponding to $2325 \mathrm{~s}$ on source

without saturating the detector. To reach the desired signal to noise ratio, we will then have to make, e.g. 23 exposures of $4 \mathrm{~min}$, which represents 2 hours on the source when taking the overheads into account.

\subsubsection{Results}

Only 3 sources have been observed and unfortunately, mostly because of bad weather, it was not possible to spend enough time on source to reach the wished signal to noise ratio. $\beta \mathrm{Lib}, \theta$ Cap and $\iota$ Cen were observed respectively for a total of 2325,5870 and 210 s on source.

Figure 1 represents some of the spectra obtained. Each spectrum has been flatfielded individually, wavelength calibrated and finally coadded to the others to create this image. The data reduction has entirely been done under the echelle context of MIDAS (Banse et al. 1983). All further data analysis concerns only the $\theta$ Cap data since it has the highest integration time, i.e. the best signal on source.

Since the Moon was $80 \%$ full during the observation, strong solar lines reflected off the Moon interferes with the observed spectrum. The best illustration is visible in Fig. 1 where at 5895.55 for $\beta$ Lib and 5895.65 for $\theta$ Cap appears a Doppler shifted solar sodium line. Most of the other lines identified in the spectrum correspond to atmospheric water contribution.

To calibrate the spectrum from the atmospheric contribution, except the mesospheric sodium, lines have been identified, fitted and suppressed. The contaminating lines to be suppressed have been identified thanks to table of atmospheric and solar lines (essentially from Lundström et al. 1991 and Moore et al. 1966). Calibration of future observations are planned to be done in two ways: observe a blank sky to determine the 


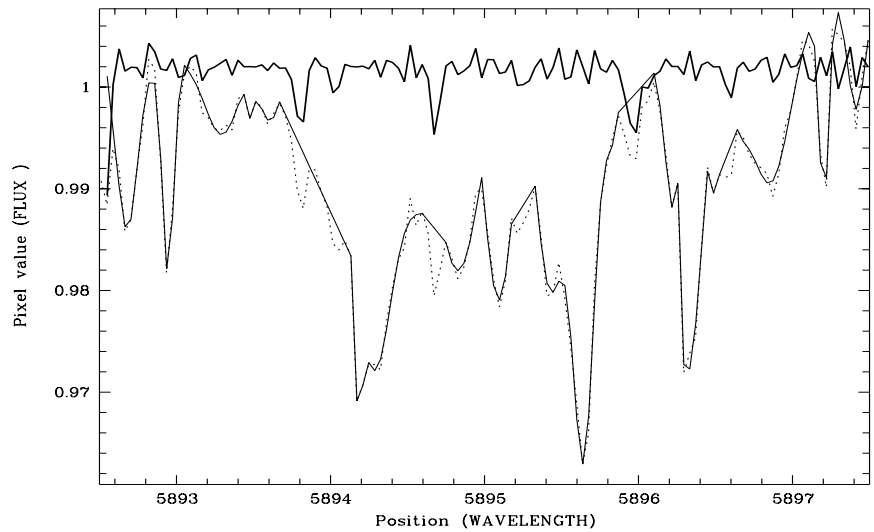

Fig. 2. Spectrum of $\theta$ Cap. The dotted black line is the original spectrum, the model fitted is represented by the thin full line; the resulting spectrum is overplotted with an artificial offset of 1 (thick full line)

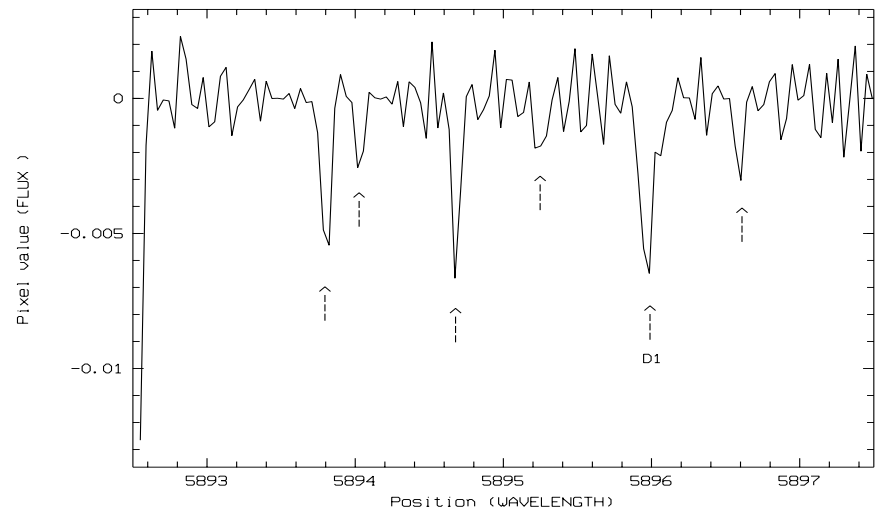

Fig. 3. Final spectrum of $\theta$ Cap, resulting from the difference between the observed spectrum and the modelled one. Except specified, arrows indicate lines that were not identified and are thus remaining. At $\sim 5896 \AA$, the arrow points at the atmospheric sodium $\mathrm{D}_{1}$ line, whose equivalent width is $0.5 \mathrm{~m} \AA$

exact position of the atmospheric lines; and compare spectra of at least 2 different sources, observed the same night, to identify the lines of solar origin thanks to their relative Doppler shift. It is also expected to be able to use a code creating a synthetic atmospheric spectrum (Lundström et al. 1991).

The identified lines were geometrically fitted with Gaussian functions and then merged into a spectrum corresponding to our model of the data (see Fig. 2). The result of the fitting is presented Fig. 3. The signal to noise depends of course on the total integration time. The noise of the background level could however be reduced by using a proper physical model to fit the identified perturbing atmospheric lines. An equivalent width of $\approx 0.5 \mathrm{~m} \AA$ at $3.5 \sigma$ was derived from the spectrum Fig. 3 .

The sodium column density, $N_{\mathrm{Na}}$, has then been derived from the formula (Spitzer 1978):

$N_{\mathrm{Na}}=W_{\mathrm{D}_{1}} /\left(8.8510^{-13} \lambda_{\mu} f_{\mathrm{D}_{1}}\right)$ where $\lambda_{\mu}=0.5896$ is the wavelength in microns, $f_{\mathrm{D}_{1}}=$ 0.32 the oscillator strength of the line and $W_{\mathrm{D}_{1}}$ the $\mathrm{D}_{1}$ line equivalent width. It resulted in $\approx 310^{9} \mathrm{~cm}^{-2}$.

\subsection{Conclusion}

Knowing the Na column density, using the theoretical prediction for sodium laser return (Milonni et al. 1998) and assuming no saturation effect in the sodium layer, one can determine the minimum laser power necessary to obtain a given magnitude laser guide star; which is our main interest in this study.

Assuming an atmospheric transmission of 0.7 , a $\sim 63 \mathrm{~W}$ projected laser power is necessary to produce a laser guide star of equivalent magnitude, $m_{V}=6$. However this calculation is true only in the case of negligible saturation, which is not the case with such a high power laser. Inverting the problem and considering a $4 \mathrm{~W}$ laser, assuming there is no saturation effect in the sodium layer at this laser power, one finds that, for a $310^{9} \mathrm{~cm}^{-2}$ sodium column density, a $m_{V}=8.74$ star can be created, which is bright enough to achieve good $\mathrm{AO}$ correction.

It is interesting to notice that the sodium column density detected in July 1998 at $29^{\circ} \mathrm{S}$ is a factor of two fainter than what was detected above Brazil $\left(23^{\circ} \mathrm{S}\right)$ in 1991 (Clemesha et al. 1992), which could simply be explained by the latitude difference. This proves the importance of such study. Following this result, ESO is planning on using some time during UVES technical nights to make similar measurements over Paranal.

Due to the dynamic range of the CCD and the minimum exposure time needed on source to reach the requested SNR, when using high resolution spectroscopy, the time resolution on the atmospheric sodium is at best 2 hours, with this type of observation. It is thus impossible to study short time scale variations of the mesospheric sodium column density. However this kind of study has to be persued over at least a period of a year to determine the "absolute" yearly minimum expected in the $\mathrm{Na}$ column density, therefore observations are planned during Chilean summer.

Among the observing facilities present at La Silla, the Coudé Echelle Spectrograph, which is fiber coupled to the ESO $3.6 \mathrm{~m}$ telescope, offers the best solution for monitoring atmospheric sodium absorption because it offers a resolution of up to 220000, which would then allow both the $\mathrm{Na} \mathrm{D}_{1}$ and $\mathrm{D}_{2}$ lines to be resolved from neighbouring water lines. This would enable a double check on the results to be made.

To conclude, spectroscopic observations are very good for statistical studies of the mesospheric sodium. The latter should primarily aim at determining the minimum amount of atmospheric $\mathrm{Na}$ one can expect for a given observing site, in order to derive the minimum laser power necessary for a performant LGS AO system. 


\section{Monitoring}

As explained previously, the mesospheric sodium has long term (seasonal) but also short term (daily or hourly) variations. The last ones cannot be studied statistically, since they are random. Once the laser power of your LGS AO system has been determined, the interest of sodium monitoring on a daily basis is:

- to detect rapid altitude and column density variations (sporadics);

- to determine the expected AO correction and thus check on the system performances;

- for queue scheduling.

In the following, the different requirements for such monitoring will be presented first, followed by a concise mention of on-going monitoring experiments.

\subsection{Instrumental requirements for a dedicated spectrograph}

The first constraint is directly linked to the sodium itself. The $\mathrm{D}_{1}$ and $\mathrm{D}_{2}$ line are respectively at $5895.94 \AA$ and $5889.773 \AA$. The last one having an hyperfine structure with a separation of $19 \mathrm{~m} \AA$.

Numerous atmospheric water vapour lines occur in the region of the spectrum around $6000 \AA$, and two lines in particular (5889.637 and 5890.09 $\AA$ ) are very close to the rest wavelength of the sodium $\mathrm{D}_{2}$ line but also much stronger. The problems associated with telluric line contamination of sodium absorption spectra have well been documented by Hobbs (1978). This implies that any instrument of interest must have a minimum resolving power of 50000 to resolve the $\mathrm{D}_{1}$ line from the closest water line. For the $\mathrm{D}_{2}$ line, the minimum resolving power requested increases to 130000 . With a resolution of one million, one is then able to resolve the hyperfine structure of the $D_{2}$ line. The other point to consider is the equivalent width of $1 \mathrm{~m} \AA$ of the atmospheric $\mathrm{Na}_{1}$ line. This means that a signal to noise of 1000 is necessary to detect it with a $15 \%$ accuracy.

Using e.g. the CES at the La Silla ESO $3.6 \mathrm{~m}$ telescope, with a resolution of 220000 , one finds that a signal to noise of 1000 can be reached in 9 min for $\beta$ car $(V=1.7)$ and in 2.5 hours for $51 \mathrm{Oph}(V=4.8)$. At best, with the brightest star, only "long term sporadics" can be studied. The short-life sporadics (few minutes at most) cannot be detected by this technique!

However, Patriarchi \& Cacciani (1999) are offering a solution to make short time integrations to monitor the mesospheric sodium layer column density with small aperture $(\approx 50 \mathrm{~cm})$ telescopes. Their system is based on a magneto-optical filter already widely used in solar observations (Cacciani et al. 1994). This filter is compact, stable in wavelengths and profile shape. It displays a very narrow spectral transmission band (down to $70 \mathrm{~m} \AA$ ) and also has a high peak transmission (up to 40\%). Presently no results of this technique have been presented.

\subsection{Existing experiments - Ongoing work}

The group of R. Angel (Tucson, Az) has been doing atmospheric $\mathrm{Na}$ measurements for several years in relation with their LGS-AO experiments at the MMT (Lloyd-Hart et al. 1995; Lloyd-Hart et al. 1998). Recent observations have been aimed at measuring the sodium column density simultaneously with the LGS visible magnitude, which has a direct bearing on the expected efficiency of the LGS adaptive optics correction. Ge et al. (1997) found an equivalent $m_{V}=10.3$ star for the LGS, using a $1 \mathrm{~W}$ projected circularly polarised sodium laser beam for an atmospheric sodium column density of $3.710^{9} \mathrm{~cm}^{-2}$. They made several successful measurements during March and May 1997, monitoring similar sodium layer patches, during the simultaneous return experiments.

The MMT experiments established the important result that linearly and circularly polarized laser returns are proportional to the simultaneous sodium column density (see Fig. 4 of Ge et al. 1998). Moreover, circularly polarized laser provides $\sim 30 \%$ increase in fluorescent return over linearly polarized laser (Ge et al. 1997).

Together with the distributed column density measurements (e.g. seasonal and diurnal variations), it is now possible to estimate laser power requirements for any specified guide star brightness, which is a crucial result, in order to determine the choice of laser type and power for the laser guide star AO systems.

Other groups related to existing or planned LGS-AO systems recognise the importance of studies as described above. Most of them are thus thinking of monitoring the mesospheric sodium but mostly using existing fully equipped nearby telescope, to realise spectro-photometric observations.

\subsection{Future possibilities}

In order to monitor the atmospheric sodium as efficiently as possible, one would like to have short exposure time. For over 30 years (Ageorges et al. 1999 and reference therein), lidar technology has been used to study the atmosphere and proved its superiority over other techniques. It gives the most information in the least time.

Presently the best solution for short measurements of the $\mathrm{Na}$ column density is via lidar technology. These can be quite portable and with a $20 \mathrm{~Hz} 100 \mathrm{~mJ}$ pulse at $590 \mathrm{~nm}$, one can record the sodium layer in about $10 \mathrm{~s}$, in clear nights, with a $2.7 \mathrm{~m}$ collector (Wuerker \& Wong 1997). A 1 m collector, would mean 
Table 1. Table summarising the impact of some characteristics of the atmospheric Na layer on LGS-AO systems and the means to study them

\begin{tabular}{|l|l|l|}
\hline LGS parameter & Atmospheric Na layer characteristics & Means of Observations \\
\hline \hline LGS power & Seasonal variations & $\begin{array}{l}\text { Statistical study } \\
\text { (spectroscopy) }\end{array}$ \\
\hline LGS focus & $\begin{array}{l}\text { Long Term variations } \\
\text { Short term variations }(<1 \mathrm{~min})\end{array}$ & $\begin{array}{l}\text { Statistical studies } \\
\text { Monitoring (lidar) }\end{array}$ \\
\hline Scheduling of observations & Daily \& hourly variations & $\begin{array}{l}\text { On-site statistics } \\
\text { Monitoring (lidar) }\end{array}$ \\
\hline
\end{tabular}

10 times longer integration time, which is still largely shorter than what is reachable presently with high resolution spectroscopic studies. Another advantage of lidar techniques is the determination of the profile of the sodium layer. The main drawback is the cost of at least few $\$ 10000$ for the laser. However "low cost" lidar experiments have started recently (Michaille et al. 2000).

The normal lidar involves transmitting short monochromatic light pulses and measuring the time-offlight of the photons (total of emitted and backscattered) in order to provide information about the scattering medium. It has been proposed to modify the Max Planck for Extraterrestrial physics ALFA (Adaptive optics with Laser For Astronomy) laser (Quirrenbach et al. 1998), at work in Calar Alto (Spain), in order to use it as a lidar. ALFA is a CW laser. It is proposed to transform it by introducing an extra-cavity acousto-optic modulator in the outgoing collimated beam. In these experiments, the $3.5 \mathrm{~m}$ telescope itself will be used as a collector. The detection will be made on a high quantum efficiency avalanche photo-diode. Each detected photon returned will be time tagged in a multi-channel scaler memory syncronised to the laser modulation.

It is important that such an instrument be designed so that the $\mathrm{Na}$ profile can be measured as quickly as possible, with minimal time lost for astrophysical observations. The final purpose is to get a world unique operating system that allows to do both LGS AO observations and simultaneously to monitor the atmospheric sodium and thus correct on-line for its variations (e.g. focus). This experiment actually took successfully place in October 1999 (Butler et al. 2000). The next step is now to use the laser launch telescope as a "lidar collector".

To summarise, presently, the Na monitoring experiments performed in conjunction with LGS-AO observations have been aimed at determining simultaneously the LGS intensity and the atmospheric Na column density in order to derive information on the LGS efficiency. This is possible because monitoring the laser power and estimating the amount of $\mathrm{Na}$ present in the atmosphere at the moment of the observations (monitoring), enable to pre- dict the return from the laser (magnitude of the LGS), only under the assumption that no saturation occurs in the sodium layer. A by-product of this study is the monitoring of the LGS-AO itself, in terms of quality of correction under known observing conditions in comparison to a theoretically perfect system.

What is currectly missing are statistical values for the variations of the centroid of the atmospheric Na height. Only these statistics would determine the real necessity of an atmospheric sodium monitor. Indeed, if the average altitude's height variations are small compared to the WFS error budget, they will then not affect the focus of the LGS. If however they are stronger, two things can happen: their frequency is higher than $0.016 \mathrm{~Hz}$, then they can be corrected by defocusing the LGS WFS; or the variations happen on timescale much smaller than 1 min, then a separate sodium monitor is really necessary to identify these periods for which the AO correction will be poorer.

\section{Conclusion}

Table 1 summarises the actual knowledge on the study of the mesospheric sodium layer for LGS-AO systems. The first column compiles the LGS parameters, and Col. 2 the "corresponding" Na characteristics. The last column indicates ways of studying these characteristics.

Spectroscopic studies like the one presented in Sect. 3 are perfectly suited for statistical studies of mesospheric sodium column density. They allow to establish the minimum Na column density and thus to derive the laser power required to produce a LGS of specified magnitude. The interest of monitoring the sodium on a daily basis, therefore, is to give an indication of the expected LGS-AO correction. A comparison with the actual correction is also a way of checking on the system performances.

Due to their adverse effect on the stability of the mesospheric sodium centroid height, it is desirable to establish the statistics of the sporadics'appearance. If, as Kwon et al. (1988), one finds a LST slot where a huge majority of these sporadics appear for the observing site, LGS-AO observations can then be programmed outside this window. It is also very important to determine the time scale of these altitude variations. Indeed, if changes in focus, due 
to sporadics, happen on time-scale bigger than $\approx 10 \mathrm{~min}$, one could then correct for it by recording the defocus on the wavefront sensor and averaging over few minutes to determine the correction needed. If however, as it seems from lidar observations, changes occur on seconds to few minutes time scale, one then needs a separate monitor to identify these periods. The previously mentioned way of correcting the defocus is not possible anymore since on short time scale one does not know how much of the defocus is due to the atmosphere and how much is linked to the motion of the sodium layer itself. Of course, what can be called the boundary between "mid-" and "short-" term variations depends on the defocus rms accepted by the AO systems used.

Acknowledgements. NUIG and ESO are part of the European Union (EU) TMR Network "Laser Guide Star for 8 m-class Telescopes" (contract No. FMRX-CT96-0094). NA is grateful to the EU for financial support. The ESO NTT team is also thanked for its support during the recent observations and especially O. Hainaut.

\section{References}

Ageorges N., Hubin N., Redfern R.M., 1999, in Astronomy with adaptive optics present results and future programs, ESO conference and workshop proceedings 56, Bonaccini D. (ed.), pp. 3-20

Banse K., et al., 1983, MIDAS - ESO's new processing system, ESO Messenger 31, 26-28

Beatty T.J., Collins R.L., Hostetler C.A., Sechrist C.F. Jr., 1989, Geophys. Res. Lett. 16, 1019

Bowman M.R., Gibson A.J., Sandford M.C.W., 1969, Nat 221, 456-457

Bricard J., Kastler A., Robley R., 1949, C.R. Acam. Sci. Paris 228, 1601-1603

Butler D.J., Davies R.I., Fews H.S., et al., 2000, SPIE 4007 (in press)

Cacciani A., et al., 1994, Theoretical and experimental study of the magneto-optical filter. Jet Propulsion Laboratory internal report D11900, Pasadena

Clemesha B.R., 1995, J. Atm. Terr. Phys. 57, 725-736

Clemesha B.R., Simonich D.M., Takahashi H., Batista P.P., Sahai Y., 1992, J. Geophys. Res. 97, 5981-5985

Clemesha B.R., Kirchhoff V.W.J.H., Simonich D.M., Takahashi H., Batista B.P., 1980, J. Geophys. Res. 85, 3480-3484

Clemesha B.R., Kirchhoff V.W.J.H., Simonich D.M., Takahashi H., 1978, Geophys. Res. Let. 5, 873-876

Cox D.P., Reynolds R.J., 1987, ARA\&A 25, 303

Davies R.I., et al., 1998, Adaptive optical system technologies, Proc. SPIE 3353, 116-124

Dekker H., Delabre B., D'Odorico S., 1986, in Instrumentation in astronomy VI, SPIE 627, 339-348

Dekker H., D'Odorico S., Fontana A., 1994, The Messenger 76, $16-20$

Donahue T.M., Blamont J.E., 1961, Ann. Geophys. 17, 116133

Gardner C.S., Senft D.C., Kwon K.H., 1988, Nat 332, 142
Ge J., et al., 1997, Mesosphere Sodium column density and laser guide star brightness. ESO workshop on Laser technology and laser guide star for adaptive optics astronomy, Proceedings \# 55, Hubin N. (ed.), pp. 10-15

Ge J., et al., 1998, Simultaneous measurements of sodium column density and laser guide star brightness, Adaptive optical system technologies, Proc. SPIE 3353, 242

Gibson A.J., Sandford M.C.W., 1971, J. Atm. Terr. Phys. 33, $1675-1684$

Gu Y.Y., Qian J., Papen G.C., Swenson G.R., Espy P.J., 1995, Geophys. Res. Lett. 22, 2805-2808

Hobbs L.M., 1978, ApJ 222, 491-507

Hubin N., Noethe L., 1991, Isoplanatic angle measurements for stars with 10 minutes of arc separation, ESO internal report

Hunten D.M., Vallance Jones A., Ellyett C.D., McLauchlan E.C., 1964, J. Atm. Terr. Phys. 26, 67-76

Jegou J.P., 1985, Ph.D. Thesis, Univ. Paris VI

Kwon K.H., Senft D.C., Gardner C.S., 1988, J. Geophys. Res. 93, 14199-14208

Lloyd-Hart, et al., 1998, ApJ 493, 950

Lloyd-Hart, et al., 1995, ApJ 439, 455-473

Lundström I., Ardeberg A., Maurice E., Lindgren H., 1991, A\&AS 91, 199-208

McNutt D.P., Mack J.E., 1963, J. Geophys. Res. 68, 3419-3429

Michaille, et al., 2000, Monitoring the variation of mesospheric sodium density at La Palma, SPIE 4007 (in press)

Milonni P.W., Fugate R.Q., Telle J.M., 1998, JOSA A 15, 217233

Moore C.E., Minnaert M.G.J., Houtgast J., 1966, The Solar Spectrum $2935 \AA$ to $8770 \AA$

Nagasawa C., Abo M., 1995, Geophys. Res. Lett. 22, 263-266

Olivier, et al., 1996, Am. Astron. Soc. Meet. 189, \#42.08

O’Sullivan C.M.M., Redfern R.M., Ageorges N., et al., 2000, Exp. Astron. (in press)

Papen G.C., Gardner C.S., Yu G., 1996, in: Adaptive Optics 13, OSA Technical Digest Series, Optical Society of America, Washington DC, p. 96

Paresce F., 1984, AJ 89, 1022

Patriarchi P., Cacciani A., 1999, A\&A 344, L-45

Quirrenbach A., Hackenberg W., Holstenberg H.C., Wilnhammer N., 1998, SPIE 3126, 35-43

Rees M.H., Romick G.J., Belon A.E., 1975, Ann. Geophys. 31, $311-320$

Rigaut F., 1997, ESO workshop on Laser technology and laser guide star for adaptive optics astronomy, Proceedings \# 55, Hubin N. (ed.), pp. 1-5

Senft D.C., Collins R.L., Gardner C.S., 1989, Geophys. Res. Lett. 16, 715-718

Simonich D.M., Clemesha B.R., Kirchhoff V.W.J.H., 1979, J. Geophys. Res. 84, 1543-1550

Slipher V.M., 1929, PASP 41, 262

Spitzer L., 1978, "Physical processes in the interstellar medium". John Wiley \& Sons, New York

von Zahn U., Hansen T.L., 1988, J. Atmos. Terr., Phys. 50, 93-104.

von Zahn U., von der Gathern P., Hansen G., 1987, Geophys. Res. Lett. 14, 76-79

Welsh, et al., 1994, ApJ 437, 638-657

Welsh, et al., 1990, ApJ 358, 473-484

Wuerker R.F., Wong A.Y., 1997, ESO workshop on Laser technology and laser guide star for adaptive optics astronomy, Proceedings \# 55, Hubin N. (ed.), pp. 201-203 